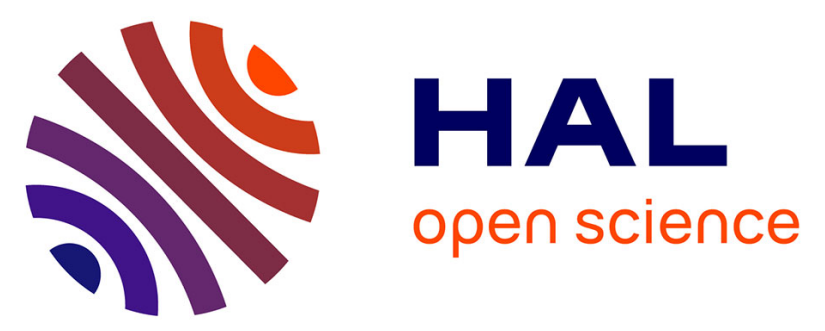

\title{
A mixed finite element method for acoustic wave propagation in moving fluids based on an Eulerian-Lagrangian description
}

Fabien Treyssede, Gwenael Gabard, Mabrouk Ben Tahar

\section{- To cite this version:}

Fabien Treyssede, Gwenael Gabard, Mabrouk Ben Tahar. A mixed finite element method for acoustic wave propagation in moving fluids based on an Eulerian-Lagrangian description. Journal of the Acoustical Society of America, 2003, 113 (2), pp 705-716. hal-00612473

HAL Id: hal-00612473

https://hal.science/hal-00612473

Submitted on 29 Jul 2011

HAL is a multi-disciplinary open access archive for the deposit and dissemination of scientific research documents, whether they are published or not. The documents may come from teaching and research institutions in France or abroad, or from public or private research centers.
L'archive ouverte pluridisciplinaire $\mathbf{H A L}$, est destinée au dépôt et à la diffusion de documents scientifiques de niveau recherche, publiés ou non, émanant des établissements d'enseignement et de recherche français ou étrangers, des laboratoires publics ou privés. 


\title{
A mixed finite element method for acoustic wave propagation in moving fluids based on an Eulerian-Lagrangian description
}

\author{
Fabien Treyssède ${ }^{\text {a) }}$, Gwénaël Gabard, and Mabrouk Ben Tahar \\ Université de Technologie de Compiègne, Laboratoire Roberval UMR 6066, \\ Secteur Acoustique, BP 20529, 60205 Compiègne Cedex, FRANCE
}

Received:

Suggested running title: "Sound propagation in non-uniform flows"

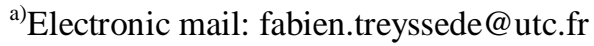




\begin{abstract}
:
A non-standard wave equation, established by Galbrun in 1931, is used to study sound propagation in non-uniform flows. Galbrun's equation describes exactly the same physical phenomenon that the linearized Euler's equations (LEE) but is derived from an Eulerian-Lagrangian description and written only in term of the Lagrangian perturbation of the displacement. This equation has interesting properties and may be a good alternative to the LEE: only acoustic displacement is involved (even in non-homentropic cases), it provides exact expressions of acoustic intensity and energy, and boundary conditions are easily expressed because acoustic displacement whose normal component is continuous appears explicitly. In this paper, Galbrun's equation is solved using a finite element method (FEM) in the axisymmetric case. With standard finite elements, the direct displacement-based variational formulation gives some corrupted results. Instead, a mixed finite element satisfying the inf-sup condition is proposed to avoid this problem. A first set of results is compared with semi-analytical solutions for a straight duct containing a sheared flow (obtained from Pridmore-Brown's equation). A second set of results concerns a more complex duct geometry with a potential flow and is compared to results obtained from a multiple-scale method (which is an adaptation for the incompressible case of a Rienstra's recent work).
\end{abstract}

PACS numbers: 43.20.Bi, 43.28.Py, 43.20.Mv 


\section{INTRODUCTION}

Propagation of acoustic disturbances in non-uniform flows is a subject of great interest in many practical problems, particularly in transport engineering with automotive exhaust systems, aeronautical turbofan engine inlet ducts, etc. The understanding of this phenomenon is a central feature for the prediction of noise and for designing components that efficiently attenuate sound. In practice, the shape of these components is often complex and flows are not uniform. Thus, the basic equations that describe such a problem must be able to cope with those complexities. Two kinds of formulations are mainly used: the linearized Euler's equations (LEE) and the fullpotential formulation. However as discussed further below, there exists another wave equation, which is a reformulation of the LEE.

The full-potential formulation is obtained from the LEE by assuming both flow and disturbances irrotationality. Thus, it constitutes a specific case of the general LEE. The corresponding propagation equation is scalar and written only in term of the acoustic velocity potential. This makes its resolution far easier and explains why it is much more widespread in the litterature. On the basis of the full-potential equation, some authors studied the effect of flow variation and multidirectionality upon sound propagation in ducts with variable cross-sections, using a finite element method $(\mathrm{FEM})^{1,2,3}$ or a boundary element method ${ }^{4}$. Besides, the analysis of pure propagation phenomena has 
naturally been extended to radiation by many authors by the use of various techniques (FEM combined with a boundary element method ${ }^{5,6,7,8,9}$ or a wave-envelope element technique ${ }^{10,11,12,13}$, dual reciprocity boundary element method $^{14}$, etc.).

Recently, Rienstra ${ }^{15}$ developed an analytical model based on a multiple-scales method to study pure propagation in slowly varying cross-section ducts. Rienstra and Eversman $^{16}$ made comparisons between the multiple-scales and FEM solutions that validates both models (though it highlights the limits of a multiple-scales method when reflections or conversions into other modes occur). In this paper, Rienstra's analytical model is used to give a reference solution that validates the FEM implementation of Galbrun's equation.

Because of its relative simplicity, the full-potential equation is a powerful formulation and may be sufficient to study sound propagation in flows. However, its main drawback is that it cannot take into account rotational mean flows. For instance, flow rotationality cannot be neglected when the effect of boundary layer refraction is important (see, for instance, Ref. 17,18,19) or when a mean flow swirl is present (this typically happens behind a rotor stage - see Ref. 20).

Actually, if the mean flow is rotational, the decomposition of the perturbations in terms of independent acoustic and rotational modes is no longer valid. The LEE must be directly solved. These equations represent a system of five equations and five unknowns, 
which can be reduced to four if the flow is supposed to be homentropic (pressure and density are then directly related). Given the complexity of the system, some attempts were made to simplify its solution. For instance, Nayfeh et al. ${ }^{21}$, Uenishi and Myers ${ }^{22}$ developed a numerical method for solving acoustic propagation in ducts with variable cross-sections and mean sheared flow.

In the late 1970s, Abrahamson ${ }^{23}$, Astley and Eversman ${ }^{24,25}$ implemented the direct LEE respectively for axisymmetric and 2D geometries, using a FEM. Good results were presented for simple cases but unfortunately, their work has not been continued. Astley and Eversman ${ }^{26}$ also outlined the existence of spurious modes when using a FEM to formulate the eigenvalue problem for a straight lined duct with shear flow.

More recently, Golubev and Atassi ${ }^{27}$ studied a straight duct containing a mean flow with swirl and showed the coupling that occurs between acoustic and rotational modes. Cooper and Peake ${ }^{20}$ extended Golubev's study to slowly varying lined ducts by applying a multiple-scales method. Results showed the influence of the mean flow swirl, i.e. corotating modes are always much more damped than those in a non-swirling flow and counter-rotating modes may be amplified.

Furthermore, it must be noticed that the LEE have recently been implemented by several authors (see, for instance, Ref. 28,29,30) using numerical methods based on finite difference schemes. These references proved the operator ability to adequately 
propagate sound wave but, so far, numerical applications are still limited to simple geometries because of numerical difficulties and high computer resources requirement.

Through what is cited above, it can be seen that the use of the LEE may be essential and inescapable in several practical cases. However, this system is far more complex than the scalar full-potential equation, and it is thus rarely solved for general cases. In this paper, it is attempted to develop a general method based on a FEM to solve sound propagation problems with rotational mean flows. Instead of choosing the LEE, a nonstandard wave equation originally established by Galbrun ${ }^{31}$ in the early 1930s is considered. As explained in the following section, this equation is derived from an Eulerian-Lagrangian description and is an exact reformulation of the LEE. Thus, Galbrun's equation a priori describes exactly the same physical phenomenon. Its main feature is that it constitutes a second-order linear partial differential equation written only in terms of the displacement perturbation.

Although only few works deal with this equation, it may be an interesting alternative to the LEE. Only acoustic displacement is involved (even in non-homentropic cases), which yields a gain of one to two unknowns compared to the LEE ; it also provides exact expressions of acoustic intensity and energy ; besides, boundary conditions are easily expressed because acoustic displacement (whose normal component is generally continuous at any interface between two media) appears explicitly, which avoid the somewhat difficult use of Myers' condition ${ }^{32}$. 
In 1985, Poirée ${ }^{33}$ detailed the Eulerian-Lagrangian description in order to derive Galbrun's equation and its extension to nonlinear problems. He also derived some general continuity conditions valid at any straight interface. Godin ${ }^{34}$ independently obtained Galbrun's equation in a quite different way. In particular, he derived exact expressions of acoustic energy and intensity, and boundary conditions for free, rigid, and absorbing surfaces in terms of the displacement. Ben Tahar and Goy ${ }^{35}$ developed a variational formulation to study vibroacoustic problems with mean flow.

Recently, Peyret and Elias ${ }^{36}$ proposed a direct displacement-based formulation of Galbrun's equation solved using a FEM. They also derived the same energy conservation law as Godin but with a different approach. Bonnet et al. ${ }^{37}$ pointed out the fact that the direct displacement-based formulation associated with Galbrun's equations does not necessarily converge with standard finite elements and proposed a method to regularize the variational formulation in the case of a uniform flow. These two last references are discussed in detail later (see Sec. III).

In this paper, a mixed variational formulation based on the pressure-displacement variables is presented in order to avoid some spurious solutions. Though the overall method is quite general, finite element discretization and numerical results are presented for the axisymmetric case. A first set of results consists in comparing FEM with semianalytical solutions for a straight duct containing a sheared flow (obtained from 
Pridmore-Brown equation). A second set of results concerns a more complex duct geometry with a potential flow and is compared to results obtained from a multiple-scale method (which is an adaptation of Rienstra's work for the incompressible case - see Ref. $15)$.

\section{THEORY}

Compared to the classical Eulerian description, the Eulerian-Lagrangian description is not usual. This section briefly recalls the latter before giving Galbrun's equation and the associated expression of acoustic intensity. This section does not give details about calculations but reviews can be found in Ref. 31,33,34.

\section{A. The Eulerian-Lagrangian description}

In the Eulerian description, perturbations are Eulerian and expressed with Eulerian variables. This description is implicitly used when Euler's equations are directly linearized. The Eulerian-Lagrangian description consists in considering Lagrangian perturbations of quantities expressed in terms of Eulerian variables. In order to give a physical comprehension of what Lagrangian and Eulerian perturbations mean, the perturbed (or total) and non-perturbed (or mean flow) states have to be described first. 
Define the geometrical position $\mathbf{x}$ of a given particle in the mean flow configuration and its position $\mathbf{y}$ in the perturbed configuration. Then, if $\mathbf{w}^{\mathbf{L}}$ denotes the displacement perturbation of this particle, $\mathbf{x}$ and $\mathbf{y}$ are related by:

$$
\mathbf{y}=\mathbf{x}+\varepsilon \mathbf{w}^{\mathbf{L}}
$$

In the remainder of this paper, $\Psi$ represents any physical quantity (tensor of arbitrary order) and the subscript 0 is used to distinguish mean flow quantities from their total (or perturbed) counterpart. Then, two kinds of perturbations can be defined:

$$
\begin{aligned}
& \varepsilon \Psi^{E}=\Psi(\mathbf{y}, t)-\Psi_{0}(\mathbf{y}, t) \\
& \varepsilon \Psi^{L}=\Psi(\mathbf{y}, t)-\Psi_{0}(\mathbf{x}, t)
\end{aligned}
$$

Superscripts E and L denote respectively Eulerian and Lagrangian perturbations. From these definitions, it can be seen that Eulerian perturbations are associated with the same geometrical point but not the same particle, whereas Lagrangian perturbations are associated with the same particle. Using Eq. (2.1) into Eq. (2.2), a Taylor expansion up to the first order gives the fundamental relationship between Eulerian and Lagrangian perturbations:

$$
\Psi^{L}=\Psi^{E}+\mathbf{w}^{\mathbf{L}} \cdot \nabla \Psi_{0}
$$

As stated earlier, $\Psi$ represents any physical quantity and the above equation holds for pressure fluctuations, density, velocity, etc. 


\section{B. Galbrun's equation}

Equation (2.3) is now applied to pressure, density and velocity perturbations. This yields:

$$
\begin{aligned}
& p^{L}=p^{E}+\mathbf{w}^{\mathbf{L}} \cdot \nabla p_{0}, \quad \rho^{L}=\rho^{E}+\mathbf{w}^{\mathbf{L}} \cdot \nabla \rho_{0}, \\
& \frac{d \mathbf{w}^{\mathbf{L}}}{d t}=\mathbf{v}^{\mathbf{E}}+\mathbf{w}^{\mathbf{L}} \cdot \nabla \mathbf{v}_{\mathbf{0}}
\end{aligned}
$$

where $d / d t=\partial / \partial t+\mathbf{v}_{\mathbf{0}} . \nabla$ is the material derivative. Equations (2.4) allow to express every Eulerian perturbations in terms of Lagrangian perturbations. Replacing every fluctuation by its Lagrangian counterpart into the LEE leads (after tedious calculations) to Galbrun's equation:

$$
\rho_{0} \frac{d^{2} \mathbf{w}^{\mathbf{L}}}{d t^{2}}-\nabla\left(\rho_{0} c_{0}^{2} \nabla \cdot \mathbf{w}^{\mathbf{L}}\right)+\left(\nabla \cdot \mathbf{w}^{\mathbf{L}}\right) \nabla p_{0}-{ }^{\mathrm{T}} \nabla \mathbf{w}^{\mathbf{L}} \cdot \nabla p_{0}=0
$$

and the Lagrangian density perturbation is explicitly given by:

$$
\rho^{L}=-\rho_{0} \nabla \cdot \mathbf{w}^{\mathbf{L}}
$$

Eq. (2.6) constitutes the mass continuity equation obtained from a Lagrangian-Eulerian description. It simply states that density fluctuations are balanced by dilatation fluctuations.

In order to derive Galbrun's equation, perfect fluid and isentropic assumptions have implicitly been made by starting from the LEE. For Lagrangian perturbations, the isentropic assumption leads to the well-known pressure-density relationship:

$$
p^{L}=\rho^{L} c_{0}^{2}
$$


which yields the following explicit equation for the Lagrangian pressure:

$$
p^{L}=-\rho_{0} c_{0}^{2} \nabla \cdot \mathbf{w}^{\mathbf{L}}
$$

Some important remarks should now be adressed. Unlike Eulerian perturbations, Eq. (2.7) remains valid even for inhomogeneous media and/or for non-homentropic flows. In these situations, solving the LEE would require an additional equation, that is the linearized energy equation (see, for instance, Ref. 38). In fact, for Lagrangian perturbations, Eq. (2.7) still holds because the equation of state applies for a given particle so that the thermodynamical system is closed.

Thus, Galbrun's equation obviously provides a first advantage (compared to the LEE) because this equation is expressed with the Lagrangian displacement as a unique unknown, which yields a gain of one to two variables.

Another asset is the existence (but not uniqueness) of a Lagrangian density associated with Galbrun's equation (see Ref. 36). This yields an exact energy conservation law and exact expressions for the acoustic energy and intensity. These expressions can be found in Ref. 34 and 36. In particular, the acoustic intensity is given by:

$$
\mathbf{i}=\rho_{0}\left(\frac{\partial \mathbf{w}^{\mathbf{L}}}{\partial t} \cdot \frac{d \mathbf{w}^{\mathbf{L}}}{d t}\right) \mathbf{v}_{\mathbf{0}}+\left(p^{L}-\mathbf{w}^{\mathbf{L}} \cdot \nabla p_{0}\right) \frac{\partial \mathbf{w}^{\mathbf{L}}}{\partial t}
$$


It can be noticed that the Lagrangian displacement inevitably appears in this expression, which likely explains why no exact formulation for the intensity has been found based on a pure Eulerian description.

\section{NUMERICAL METHOD}

In this section, Galbrun's equation is solved using a FEM. From now on, fluctuations are assumed to have an $e^{-i \omega t}$ time dependence. Besides, the mean flow is steady and the mean pressure is supposed to be constant for simplicity (dropping the last two terms of Eq. (2.5)). This assumption is not valid for aeroacoustic problems but our purpose is to solve Galbrun's equation with a FEM (as outlined by Peyret and Elias ${ }^{36}$, terms with $p_{o}$ do not present a major interest from a numerical point of view). Under these assumptions, Eq. (2.5) becomes:

$$
-\rho_{0} \omega^{2} \mathbf{w}^{\mathbf{L}}-2 i \omega p_{0} \mathbf{v}_{\mathbf{0}} \cdot \nabla \mathbf{w}^{\mathbf{L}}+\rho_{0} \mathbf{v}_{\mathbf{0}} \cdot \nabla\left(\mathbf{v}_{\mathbf{0}} \cdot \nabla \mathbf{w}^{\mathbf{L}}\right)-\rho_{0} c_{0}^{2} \nabla\left(\nabla \cdot \mathbf{w}^{\mathbf{L}}\right)=0
$$

The first subheading of this section gives a brief review about the numerical difficulties of the direct displacement based formulation associated to (3.1). In the second subheading, a displacement-pressure based mixed formulation is proposed to overcome these difficulties. A third one deals with boundary conditions and the last subheading gives some important details about the finite element discretization of the mixed formulation. 


\section{A. Displacement based formulation}

Equation (3.1) is multiplied by a trial field $\mathbf{w}^{*}$ and integrated over the domain $\Omega$. Then, integrating by part the last two terms (which imply second order derivatives) and half the second term yields the following direct displacement based variational formulation:

$$
\begin{aligned}
& \int_{\Omega} \rho_{0} c_{0}^{2}\left(\nabla \cdot \mathbf{w}^{*}\right)\left(\nabla \cdot \mathbf{w}^{\mathbf{L}}\right) d \Omega-\omega^{2} \int_{\Omega} \rho_{0} \mathbf{w}^{*} \cdot \mathbf{w}^{\mathbf{L}} d \Omega \\
& \quad-i \omega \int_{\Omega} \rho_{0} \mathbf{w}^{*} \cdot\left(\mathbf{v}_{\mathbf{0}} \cdot \nabla \mathbf{w}^{\mathbf{L}}\right) d \Omega+i \omega \int_{\Omega} \rho_{0}\left(\mathbf{v}_{\mathbf{0}} \cdot \nabla \mathbf{w}^{*}\right) \cdot \mathbf{w}^{\mathbf{L}} d \Omega \\
& \quad-\int_{\Omega} \rho_{0}\left(\mathbf{v}_{\mathbf{0}} \cdot \nabla \mathbf{w}^{*}\right) \cdot\left(\mathbf{v}_{\mathbf{0}} \cdot \nabla \mathbf{w}^{\mathbf{L}}\right) d \Omega \\
& \quad+\int_{S} \mathbf{w}^{*} \cdot\left\{\rho_{0}\left(\mathbf{v}_{\mathbf{0}} \cdot \mathbf{n}\right) \frac{d \mathbf{w}^{\mathbf{L}}}{d t}+p^{L} \cdot \mathbf{n}\right\} d S=0 \quad \forall \mathbf{w}^{*}
\end{aligned}
$$

It can be noticed that the above formulation is presented in such a way that the first line corresponds to the no-flow case and the second and third one to the presence of mean flow (the last line is a boundary integral).

This formulation is used in Ref. 36. However, when standard finite elements are implemented to discretize the formulation, solutions are generally corrupted, even in the no-flow case. This phenomenon is purely numerical. An example of spurious solution is given in Sec. IVA. 
Bonnet \& al. ${ }^{37}$ have recently proposed a regularized formulation of Galbrun's equation in the uniform flow case (with this method, some specific terms are added in the formulation (3.2)). A good convergence is obtained but limitations of the method arise for the generalization to arbitrary mean flows.

The no-flow case was first studied in the 1970s when considering vibrations of coupled fluid-structure systems (see, for instance, Ref. 39) and was proved to exhibit spurious circulation modes with non-zero frequencies. Basically, this phenomenon is due to a bad accuracy of the divergence and curl (calculated from derivatives of displacements), which in turn affects the displacement prediction itself. In order to cope with this numerical phenomenon, several methods have been proposed, such as the penalty method ${ }^{39}$, edge finite element ${ }^{40}$, and mixed finite element $\operatorname{method}^{41,42}$.

In the presence of mean flow, the penalty method and edge finite element method cannot be directly applied because the displacement field is generally no more irrotational. Thus, the method chosen in this paper is naturally based on a mixed finite element formulation. To conclude this subheading, one emphasizes that the overall problem in the now-flow case is typically analogous to incompressible elasticity or fluid $^{43}$ and electromagnetics ${ }^{44}$, and is often referred to as "locking" in the litterature. 


\section{B. Mixed formulation}

A mixed variational formulation based on pressure-displacement variables is now derived. Although pressure is now an explicit unknown of Galbrun's equation (as in the LEE), the efficiency of such a formulation to prevent corruption by spurious solutions has already been demonstrated by Wang and Bathe ${ }^{42}$ in the no-flow case when considering fluid-structure interactions. It was also extensively and rigorously analyzed by Brezzi and Fortin ${ }^{45}$ in a general way (see also Bathe ${ }^{43}$ for a more engineering-oriented approach applied to incompressible elasticity).

In the presence of arbitrary mean flow, an analogous mixed formulation of Ref. 42 can be obtained by replacing the last term of Eq. (3.1) with the pressure gradient (use of Eq. (2.8) is made). It yields the following system:

$$
\left\{\begin{array}{l}
-\rho_{0} \omega^{2} \mathbf{w}^{\mathbf{L}}-2 i \omega p_{0} \mathbf{v}_{\mathbf{0}} \cdot \nabla \mathbf{w}^{\mathbf{L}}+\rho_{0} \mathbf{v}_{\mathbf{0}} \cdot \nabla\left(\mathbf{v}_{\mathbf{0}} \cdot \nabla \mathbf{w}^{\mathbf{L}}\right)+\nabla p^{L}=0 \\
p^{L}+\rho_{0} c_{0}^{2} \nabla \cdot \mathbf{w}^{\mathbf{L}}=0
\end{array}\right.
$$

Multiplying both equations by trial fields $\mathbf{w}^{*}$ and $p^{*}$ respectively, integrating over the domain $\Omega$ and then by parts gives the following mixed variational formulation: 


$$
\begin{aligned}
& -\int_{\Omega} \frac{1}{\rho_{0} c_{0}^{2}} p^{*} p^{L} d \Omega+\int_{\Omega} \nabla p^{*} \cdot \mathbf{w}^{\mathbf{L}} d \Omega+\int_{\Omega} \mathbf{w}^{*} \cdot \nabla p^{L} d \Omega-\omega^{2} \int_{\Omega} \rho_{0} \mathbf{w}^{*} \cdot \mathbf{w}^{\mathbf{L}} d \Omega \\
& -i \omega \int_{\Omega} \rho_{0} \mathbf{w}^{*} \cdot\left(\mathbf{v}_{\mathbf{0}} \cdot \nabla \mathbf{w}^{\mathbf{L}}\right) d \Omega+i \omega \int_{\Omega} \rho_{0}\left(\mathbf{v}_{\mathbf{0}} \cdot \nabla \mathbf{w}^{*}\right) \cdot \mathbf{w}^{\mathbf{L}} d \Omega \\
& -\int_{\Omega} \rho_{0}\left(\mathbf{v}_{\mathbf{0}} \cdot \nabla \mathbf{w}^{*}\right) \cdot\left(\mathbf{v}_{\mathbf{0}} \cdot \nabla \mathbf{w}^{\mathbf{L}}\right) d \Omega \\
& +\int_{S} \mathbf{w}^{*} \cdot\left\{\rho_{0}\left(\mathbf{v}_{\mathbf{0}} \cdot \mathbf{n}\right) \frac{d \mathbf{w}^{\mathbf{L}}}{d t}\right\} d S-\int_{S} p^{*}\left(\mathbf{w}^{\mathbf{L}} \cdot \mathbf{n}\right) d S=0 \quad \forall\left\{\mathbf{w}^{*}, p^{*}\right\}
\end{aligned}
$$

where $S$ is the surface enclosing the acoustic domain $\Omega$ and $\mathbf{n}$ is the outward normal.

As in Eq. (3.2), the first line of the mixed formulation (3.4) represents the no-flow operators. These operators are almost identical to those used by Wang and Bathe ${ }^{42}$ in their mixed formulation. The only slight difference is that one has chosen to integrate by parts the second term of the second equation of system (3.3) (instead of the last term of the first equation) in order to let the normal displacement appear explicitly at the boundary.

Normal displacement at walls can thus be easily imposed (the second surface integral of Eq. (3.4) simply disappear for perfectly rigid walls). Besides, for fluidstructure interactions (not considered in this paper), normal displacement continuity could also be easily imposed by replacing the fluid normal displacement with the structure normal displacement.

In order to show the efficiency of a mixed formulation, a comparison with the displacement based formulation is given in Sec. IVA. 


\section{Boundary conditions}

Boundary conditions associated with Galbrun's equation must be carefully applied. Figure 1 represents a typical problem of propagation inside a duct carrying flow. Two types of boundary conditions must be distinguished: those imposed at walls (boundary $S_{1}$ ) and those imposed inside the fluid (boundary $S_{2}$ ).

At walls, the first surface integral of (3.4) always vanishes because the mean flow normal velocity is zero. For rigid walls, normal displacement also equals zero and the second integral also vanishes. For an absorbing wall, the adequate boundary condition is obtained from the normal displacement fluid-wall continuity. Given the wall impedance $Z$, this condition is simply given by the following relationship:

$$
\left.p^{L}\right|_{S_{1}}=-i \omega Z \mathbf{w}^{\mathbf{L}} \cdot \mathbf{n}
$$

which is simply applied by replacing the normal displacement in the second boundary integral. The fact that displacement is an explicit variable in the proposed formulation makes the impedance condition (3.5) simpler to implement than in the LEE case, that would have required the use of Myer's condition ${ }^{32}$.

When an arbitrary mean flow is present, the displacement field may not be irrotational. Thus, it is obvious that a fixed pressure inside the fluid (on $S_{2}$ ) is not 
sufficient to determine a unique solution. For instance, applying pressure (which represents the displacement divergence from Eq. (2.8)) at the duct inlet and outlet means that the rotational part of the displacement is left free. Consequently, one must impose the total displacement field everywhere a boundary condition is required inside the fluid (typically at the duct inlet-outlet). This condition is explicitly given by:

$$
\left.\mathbf{w}^{\mathbf{L}}\right|_{S_{2}}=\overline{\mathbf{w}}
$$

and is directly enforced at nodes as a constraint in the FEM model. This makes the first surface integrals in (3.4) vanish because $\mathbf{w}^{*} \equiv \delta \mathbf{w}=0$ on $S_{2}$ (i.e. forced boundary condition). Note that in many instances, it is difficult to specify the particle displacement on both surfaces labeled $S_{2}$ because only the incident component is known. A boundary condition based on a multi-modal decomposition technique overcomes this difficulty.

As a side remark, in the no-flow case, a surface with fixed pressure inside the fluid is sufficient to uniquely determine the solution because displacement is implicitly irrotational.

\section{Finite element discretization}

It has been proven that interpolations for displacement and pressure must be adequately chosen. The choice of a mixed formulation is not sufficient to avoid locking, 
and one also has to choose appropriate interpolation functions. Based on Ref. 43 and 45, this subheading gives some details about what kind of finite elements has to be used.

If a bad choice of interpolating functions is made, the element may lock and/or give some spurious pressure modes. A criterion that ensures convergence and stability of the finite element is given by the so-called inf-sup condition. Though not necessary, this condition is a strong guaranty of reliability. Details about the inf-sup condition are not given in this paper but can be found in Ref. 43 and 45. For a more practical use, a numerical test of this condition has also been developed by Chapelle and Bathe ${ }^{46}$. One example of triangular 2D-element satisfying the inf-sup condition is given on Fig. 2. This element may be referred to as the " $P_{l}^{+}-P_{l}$ ", "4-3c" or "MINI" element ${ }^{43,45}$ and is the one used in this paper. An example of higher order element is also given on the same figure (element "9-4c"). Note that some other 2D (or 3D) elements can be found in Ref. 43,46 .

In the no-flow case, elements that satisfy the inf-sup condition have successfully been implemented by Wang and Bathe ${ }^{42}$. The originality of Galbrun's equation is that the pressure-displacement relationship given by (2.8) is not altered by the presence of flow and is strictly identical to the no-flow case. This enables to directly apply the infsup condition to a Galbrun-based formulation. Thus, under the assumption that the additional operators introduced by the presence of flow (terms in $\mathbf{v}_{\mathbf{0}} . \nabla$ ) does not alter 
convergence properties of elements satisfying the inf-sup condition, it is expected that the proposed mixed FEM for solving Galbrun's equation is robust.

When flow is present, it should be noticed that the mass continuity equation obtained from the LEE implies material derivatives and no equation similar to Eq. (2.8) can be obtained. The inf-sup condition cannot then be directly applied when the LEE are considered. Thus, given the current knowledge about mixed formulations, Galbrun's equation seems to be more interesting from a numerical point of view.

The general variational formulation (3.4) is now restricted to axisymmetric geometries. Introducing the cylindrical coordinates, the following $\theta$-dependence is set:

$$
\left\{\begin{array}{l}
\mathbf{w}^{\mathbf{L}}(r, \theta, z)=\mathbf{w}^{\mathbf{L}}(r, z) e^{-i m \theta} \\
p^{L}(r, \theta, z)=p^{L}(r, z) e^{-i m \theta}
\end{array}\right.
$$

where the angular mode number $m$ is a parameter of the solution. The weighting functions are taken as:

$$
\left\{\begin{array}{l}
\mathbf{w}^{*}(r, \theta, z)=\mathbf{w}^{*}(r, z) e^{+i m \theta} \\
p^{*}(r, \theta, z)=p^{*}(r, z) e^{+i m \theta}
\end{array}\right.
$$

The (2D) acoustic domain $\Omega$ is chosen to be meshed with $4-3$ c elements. On the reference element, displacement and pressure variables are thus interpolated as follows:

$$
\left\{\begin{array}{l}
\mathbf{w}^{\mathbf{L}}(u, v)=(1-u-v) \mathbf{w}_{1}+u \mathbf{w}_{2}+v \mathbf{w}_{3}+(1-u-v) u v \mathbf{a} \\
p^{L}(u, v)=(1-u-v) p_{1}+u p_{2}+v p_{3}
\end{array}\right.
$$


where the subscripts $i(i=1,2,3)$ denotes the node number. It can be seen for the displacement, that the standard linear interpolation is enriched by introducing a generalized variable a. The term $(1-u-v) u v$ represents a bubble function: this polynomial is null on the three side of the triangle and thus maintains the compatibility $\left(\mathrm{C}^{0}\right.$ continuity).

Then, elements have four degrees of freedom per node, plus three internal (three for each component of $\mathbf{w}^{\mathbf{L}}$ ). However, these internal degrees of freedom can be condensed out before the element are assembled, which is attractive from a computational point of view.

After assembling and applying boundary conditions, the global discretized variational formulation yields the following algebraic system:

$$
\mathbf{K}_{\mathbf{r}} \mathbf{w}_{\mathbf{r}}=\mathbf{f}_{\mathbf{r}}
$$

$\mathbf{K}_{\mathbf{r}}$ is a $\omega$-dependent complex band matrix, unsymmetrical when flow is present. A sparse storage is chosen. For a fixed $\omega$, the unknown nodal displacement vector $\mathbf{w}_{\mathbf{r}}$ is finally obtained by using a LU decomposition.

\section{RESULTS}


In this section, the FEM numerical method is validated with two semi-analytical models. The first model corresponds to the well-known Pridmore-Brown equation, the second to Rienstra's multiple scale approximation (in the incompressible mean flow case in order to fit with the assumption of the FEM model - see Sec. III). Both models represents the propagation of a given $(m, n)$ mode in an infinite duct ( $m$ and $n$ denote respectively the angular and radial mode number).

They may be considered as complementary. Pridmore-Brown equation deals with a simple straight duct but a possibly sheared mean flow. Boundary layer effects upon propagation can thus be considered. In Rienstra's multiple-scales method, the mean flow must be potential but the duct is slowly varying, which permits to study more complex geometries.

Unlike Pridmore-Brown equation, Rienstra's model constitutes an approximation. It cannot be exact because of modal reflection and scattering that may occur in a varying duct. These limitations of a multiple-scales method have been highlighted by Rienstra and Eversman ${ }^{16}$ and are also demonstrated in this paper.

In the following, iso-pressure contours are given in modulus for all plots. Units are chosen to be in $\mathrm{Pa}$ in order not to minimize errors. The averaged intensity vector may also be given on the $\theta=0^{\circ}$ plane. Mean flow velocities are defined in Mach number $(M)$. Propagation and flow directions are also sketched in order to explicitly show if wave 
propagation is upstream or downstream. Besides, typical values of $\rho_{0}=1.2 \mathrm{~kg} \cdot \mathrm{m}^{-3}$ and $c_{0}=340 \mathrm{~m} \cdot \mathrm{s}^{-1}$ are used.

Test cases sweep a non-dimensional frequency range up to about $k R=20$ and the duct geometry is always meshed with a $\lambda / 10$ finite element length. This is the estimated criterion for a satisfactory convergence but a $\lambda / 8$ criterion may be sufficient to give good results. These criteria are yet modulated by a $\sqrt{1-M^{2}}$ factor due to the Doppler effect when flow is present.

\section{A. Validation for straight ducts (Pridmore-Brown equation)}

For FEM computations of this subheading, the methodology is as follows. The Lagrangian displacement is calculated from the Pridmore-Brown model and is then imposed at the duct inlet of the FEM model (in the remaining, the terms "inlet" and "outlet" are used from an acoustical point of view - see the direction of propagation upon each figure - and not from a mean flow point of view). A modal non-reflective boundary condition is preferred at the outlet, which is less constraining (in particular, the phase and amplitude are left free). Hence, the mode being enforced at the inlet/outlet boundaries, the FEM solution inside the duct is computed and compared to the semianalytical one. 
The non-reflective boundary condition is inspired from Peyret and Elias ${ }^{36}$. It simulates the one-way propagation and applies only for a given $(m, n)$ mode. In classical acoustics (no-flow case), one usually imposes on the cross-section an anechoic termination based on the wave impedance (i.e. the ratio of the pressure and the normal acoustic velocity). Here, an additional condition is required with the displacement variable in order to determine a unique solution. The velocity and the normal displacement which appear in the boundary integral of Eq. (3.4) are thus replaced with the following expressions:

$$
\rho_{0}\left(\mathbf{v}_{\mathbf{0}} \cdot \mathbf{n}\right) \frac{d \mathbf{w}^{\mathbf{L}}}{d t}=\mathbf{Z}_{\mathbf{n r}} \mathbf{w}^{\mathbf{L}} ; \mathbf{w}^{\mathbf{L}} \cdot \mathbf{n}=-\frac{1}{i \omega Z_{n r}} p^{L}
$$

$Z_{n r}$ is the standard modal non-reflecting impedance and $\mathbf{Z}_{\mathbf{n r}}$ can be viewed as a modal non-reflective matrix impedance. In the Pridmore-Brown model, material derivatives are simply given by $d / d t=-i\left(\omega-v_{0} k_{z}\right)$ because an $e^{i\left(k_{z} z-m \theta-\omega t\right)}$ dependence is chosen for the modal acoustic variables. This yields the following explicit expressions for the modal impedances defined by (4.1):

$$
\mathbf{Z}_{\mathrm{nr}}=-i \rho_{0} v_{0}\left(\omega-v_{0} k_{z}\right) \mathbf{I} ; Z_{n r}=\rho_{0} \frac{\left(\omega-v_{0} k_{z}\right)^{2}}{\omega k_{z}}
$$

where $v_{0}$ is the axial mean flow velocity and $k_{z}$ is the outlet modal axial wave-number, which is part of the semi-analytical solution. I denotes the identity matrix.

In this subheading, an annular straight duct is considered. The inner and outer radius are respectively 0.2 and $1 \mathrm{~m}$. Geometry with a typical mesh is shown by Fig. 3 . 
The first test case, given in Fig. 3, shows the efficiency of a mixed FEM compared to a displacement based one. A comparison between the semi-analytical, displacement based FEM and mixed FEM solutions is given for the pressure modulus. The example concerns a $(0,1)$ mode at $f=250 \mathrm{~Hz}$ propagating in a hard wall duct without flow. Solution obtained with a displacement based formulation is totally corrupted by rotational fields. These spurious solutions are small-scale artifact which are trapped within the model grid. Unfortunately, this numerical problem does not disappear at all when the mesh is refined. For a mixed FEM, results are clearly in good agreement with the semi-analytical solution.

The second test case (Fig. 4) concerns a $(8,0)$ mode propagating at $f=500 \mathrm{~Hz}$ in a perfectly rigid wall duct. This mode is calculated for $M=0$ and $M=-0.4$ (upstream propagation). Agreement between Pridmore-Brown and FEM solutions is perfect for both Mach number. This test case aims at pointing out one of the effect of convection upon cut-off frequencies, say a decrease by a $\sqrt{1-M^{2}}$ factor. Figure 4 clearly shows that without flow, the $(8,0)$ mode is cut-off whereas for $M=-0.4$, it becomes cut-on. In fact, for the duct dimensions, the $(8,0)$ mode has an exact cut-off frequency of $522.0 \mathrm{~Hz}$ without flow, decreased to $478.5 \mathrm{~Hz}$ at $M=-0.4$. This frequency is lower than $500 \mathrm{~Hz}$, which explains why the mode fully propagates along the duct. Examining the intensity plot for both Mach number gives directly the nature of modes: unlike at $M=-0.4$, the acoustic intensity vector is null at $M=0.0$ which proves that the mode is cut-off (energy 
does not propagate). For the $M=-0.4$ case, a comparison in terms of the pressure phase is also given by Figure 5, where a good agreement between solutions can be observed.

The third test case (Fig. 6) exhibits a $(10,1)$ mode at $f=1000 \mathrm{~Hz}$. Walls are lined and the impedance value is $Z=2040(1-i)$ for both inner and outer walls. The cross-section averaged Mach number is $M=-0.5$ (upstream propagation). In this example, a comparison between uniform and sheared flow is given in order to demonstrate the capability of the FEM approach to take into account refraction phenomena. The shear flow is arbitrarily chosen to have a boundary layer thickness of $10 \%(\delta=0.08 \mathrm{~m})$, with the same mass flow rate that the uniform profile (see Fig. 7 for the mean flow velocity profiles). This thickness is not realistic but voluntarily exaggerated in order to illustrate refraction. Note that flows with a boundary layer are obviously rotational and cannot be considered with the full-potential propagation equation.

As seen on Fig. 6, Pridmore-Brown and FEM solutions show satisfactory agreement. In particular, modal shape and attenuation are conserved in the FEM model. Results show a strong difference in amplitude between the uniform and sheared cases. In fact, for an upstream propagation, the mean flow velocity gradient due to the presence of the boundary layer tends to refract waves toward the center, thus yielding a weaker attenuation than in the uniform case. (This is the opposite for a downstream propagation, for which waves are refracted toward walls - see, for instance Ref. 17). Attenuation coefficient values can directly be obtained from the semi-analytical model. These 
coefficients are $5.6 \mathrm{~dB} \cdot \mathrm{m}^{-1}$ and $0.9 \mathrm{~dB} \cdot \mathrm{m}^{-1}$ respectively for the uniform and sheared cases. Thus, for the duct being considered, an error of about $9 \mathrm{~dB}$ is made at the outlet when a uniform profile is supposed.

\section{B. Validation for varying ducts (Rienstra's model)}

The test geometry taken is now varying (but the flow is restricted to be potential). This geometry is the same as in Rienstra's papers ${ }^{15,16}$ and is representative of a turbofan aircraft engine. It includes a circular-to-annular transition (a central body is thus present).

There are differences between the multiple scale method and the FEM. The FEM formulation admits the propagation of many modes (reflection and scattering are integral part of the solution). On the contrary, the multiple scale approximation lies in supposing that a single given mode is propagating in a single direction inside a duct. Hence, this kind of approximation neglects reflection and scattering into other modes, as clearly demonstrated by Rienstra and Eversman's study ${ }^{16}$. Reflection and scattering limitations of Rienstra's model are also both highlighted in this subheading by choosing adequate test cases.

The procedure used for FEM computation is not the same as previously exposed in Sec. IVA. The previous mono-modal non reflecting boundary condition cannot be used 
when reflections or scattering into other modes become significant. Instead, a modal decomposition technique such as in Ref. 16 is used. This technique consists in recasting the acoustic variables at the duct inlet and outlet via an eigenmode expansion. For FEM calculations, the method is as follows. On the inlet plane, the complex amplitude of the appropriate mode is specified via a forced boundary condition. On the outer plane, reflected mode amplitudes are set to zero, imposing a non-reflecting boundary condition. Inlet reflected and outlet transmitted mode amplitudes are unknown and part of the solution. In this paper, ten radial modes have been used for the expansion (given the cutoff frequencies of higher order modes, this is sufficient for the test cases presented in the following). Note that the eigenmode expansion must be done with hard walls for orthogonality (soft wall modes are not orthogonal). Thus, for test cases with lined walls, the FEM geometry has been extended up to $0.5 \mathrm{~m}$ both at the inlet and outlet by adding pieces of straight hard wall ducts (not shown on figures).

In the FEM model, the potential mean flow is first computed, also via a FEM solving Laplace's equation and then used for solving Galbrun's equation. Results presented in this subsection give comparisons between the solutions obtained by Rienstra's multiple-scales method (mono-modal) and the FEM model proposed in the present paper (FEM solutions of Rienstra and Eversman are not shown).

The first test case is depicted in Fig. 8, where geometry, mesh and mean flow are presented. At the inlet and outlet, the axial local Mach number values are $M=0.3$ and 
0.25 respectively. It must be observed that the outer wall is straight, which constitutes a slight difference with Rienstra's geometry (considered in the second test case). This essentially aims at minimizing reflections due to the outer wall.

In this first set of results (Fig. 9), a downstream $(1,1)$ mode propagating in a rigid wall duct is considered. Computations are achieved for three frequencies. At 300 and $360 \mathrm{~Hz}$, a very good agreement is obtained between FEM and multiple-scales solutions, which validates the FEM code. At these frequencies, the only cut-on modes with $m=1$ are $(1,0)$ and $(1,1)$. Other cut-on modes with $m \neq 1$ are not considered by the FEM model because $\mathrm{m}$ is a fixed parameter in the code. Consequently, the good convergence between both models demonstrates that scattering into the $(1,0)$ mode as well as reflection are almost negligible.

However, when the frequency $f=420 \mathrm{~Hz}$ is reached, a strong difference is observed. This disagreement is likely explained by partial scattering into the $(1,2)$ mode. In fact, analyzing local cut-off frequencies shows that this mode is cut-on at the outlet (its local cut-off frequency is $416.8 \mathrm{~Hz}$ ). Hence, it can be deduced from the difference observed that for $f=420 \mathrm{~Hz}$, the multiple-scales approximation fails.

The last test case (see Fig. 10) concerns a $(7,0)$ mode at $f=500 \mathrm{~Hz}$ propagating into the exact Rienstra's geometry. The outer wall is lined, the impedance value is $Z=4080(1+i)$. The central body is left perfectly rigid. Mesh and flow are not shown for 
conciseness ( $M=0.5$ and 0.49 at the inlet and outlet respectively). The goal of this example is to outline the reflection phenomenon that limits the use of a multiple-scales method. The frequency of $f=500 \mathrm{~Hz}$ is chosen in order for the $(7,0)$ mode to be the only cut-on mode. Calculations effectively give a maximum local cut-off frequency along the duct of $411.2 \mathrm{~Hz}$ for this mode and a minimum cut-off frequency of $558.6 \mathrm{~Hz}$ for the $(7,1)$ mode. This indicates that the $(7,0)$ mode is always cut-on and the $(7,1)$ always cut-off. This permits to avoid any significant scattering into other modes and thus to focus on auto-reflection of the $(7,0)$ mode only. Computations are made for both downstream and upstream propagation.

In the downstream case, a good agreement is obtained. This shows that only few reflections are produced inside the duct for this direction of propagation. In the upstream propagation case, some differences occur (on this plot, wave is propagating from the top to the bottom). Some wiggles appear and iso-pressure contours are not totally smooth. At the acoustic outlet (bottom), it can be seen on plots that the attenuation obtained by the FEM is a little greater than the semi-analytical one, which tends to prove that reflections of the $(7,0)$ mode on itself are not negligible. This may be attributed to the central body as well as the abrupt change of outer radius located at the acoustic inlet, both viewed as a narrowing for an upstream propagation.

Finally, it may be interesting to look at the acoustic intensity vector that has been plotted for both the upstream and downstream case. Because the lining of the wall 
absorbs some energy, intensity is not exactly parallel to the wall but penetrates into it. This is more visible in the upstream case, which is coherent with the fact that the downstream wave is less attenuated.

\section{CONCLUSION}

In this paper, a mixed FEM based on Galbrun's equation has been proposed to solve sound wave propagation in arbitrary flows. Compared to the LEE, this equation may have several advantages. From a theoretical point of view, it yields an exact expression for the acoustic intensity and simpler boundary conditions (especially in case of absorbing walls). From a numerical point of view, Galbrun's equation allows the direct application of the inf-sup condition, already encountered in the study of mixed FEM for incompressible media.

Results obtained with the proposed mixed FEM have been compared with two complementary semi-analytical models and have been found to be in very good agreement. The comparison with the Pridmore-Brown equation has proven the efficiency of the numerical method to take into account convection and refraction from a boundary layer, which cannot be considered with a full-potential formulation. Comparisons with a multiple-scales method have fully validated the FEM for complex geometry and have 
also confirmed limitations of a mutliple-scales approach when some significant reflection or diffraction occur.

Those results show that a mixed FEM method based on Galbrun's equation could be an interesting alternative to a finite-difference method based on the LEE, for solving aeroacoustic problems.

1 R. K. Sigman, R. K. Majjigi, and B. T. Zinn,. 'Determination of turbofan inlet acoustics using finite elements," American Institute of Aeronautics and Astronautics Journal 16, 1139-1145 (1978).

2 R. Fuller, and D. A. Bies, 'The effects of flow on the performance of a reactive acoustic attenuator," Journal of Sound and Vibration 62, 73-92 (1979).

3 Cabelli, "The influence of flow on the acoustic characteristics of a duct bend for higher order modes - a numerical study," Journal of Sound and Vibration 82, 131149 (1982).

4 Z. L. Ji, Q. Ma, and Z. H. Zhang, "A boundary element scheme for evaluation of four-pole parameters of ducts and mufflers with low mach number non-uniform flow," Journal of Sound and Vibration 185, 107-117 (1995).

5 S. J. Horowitz, R. K. Sigman, and B. T. Zinn, "An iterative finite element-integral technique for predicting sound radiation from turbofan inlets in steady flight," American Institute of Aeronautics and Astronautics Journal 24, 1256-1262 (1986). 

JT15D turbofan engine," Journal of Vibration, Acoustics, Stress, and Reliability in Design 106, 405-413 (1984).

7 R. J. Astley, and J. G. Bain, "A three-dimensional boundary element scheme for acoustic radiation in low mach number flows," Journal of Sound and Vibration 109, 445-465 (1986).

8 M. Ben Tahar, 'Formulation variationnelle par équations intégrales pour le rayonnement acoustique en présence d'écoulement non-uniforme” ("Variational formulation for acoustic radiation in non uniform flows using a boundary element method'), Thèse de docteur d'état, Université de Technologie de Compiègne (1991).

9 P. Zhang, T. Wu, and L. Lee, "A coupled FEM/BEM formulation for acoustic radiation in a subsonic non-uniform flow," Journal of Sound and Vibration 192, 333347 (1996).

10 R. J. Astley, "A finite element wave envelope formulation for acoustic radiation in moving flows," Journal of Sound and Vibration 103, 471-485 (1985).

11 Danda Roy, and W. Eversman, 'Improved finite element modeling of the turbofan engine inlet radiation problem," Journal of Vibration and Acoustics 117, 109-115 (1995).

12 W. Eversman, and D. Okunbor, "Aft fan duct acoustic radiation," Journal of Sound and Vibration 213, 235-257 (1998).

13 W. Eversman, 'Mapped infinite wave envelope elements for acoustic radiation in a uniformly moving medium," Journal of Sound and Vibration 224, 665-687 (1999). 
L. Lee, T.W. Wu, and P. Zhang, "A dual-reciprocity method for acoustic radiation in a subsonic non-uniform flow," Engineering Analysis with Boundary Elements 13 365-370 (1994).

15 S. W. Rienstra, 'Sound transmission in slowly varying circular and annular lined ducts with flow,"Journal of Fluid Mechanics 380, 279-296 (1999).

16 S. W. Rienstra, and W. Eversman, "A numerical comparison between the multiplescales and finite-element solution for sound propagation in lined flow ducts,"Journal of Fluid Mechanics 437, 367-384 (2001).

17 P. Mungur, and H. E. Plumbee, 'Propagation and attenuation of sound in a softwalled annular duct containing a sheared flow,”NASA SP-207, 305-327 (1969).

18 H. Nayfeh, J. E. Kaiser, and D. P. Telionis, "Acoustics of aircraft engine-duct systems," American Institute of Aeronautics and Astronautics Journal 13, 130-153 (1975).

19 N. K. Agarwall, and M. K. Bull, 'Acoustic wave propagation in a pipe with fully developed turbulent flow,' Journal of Sound and Vibration 132, 275-298 (1989).

20 J. Cooper, and N. Peake, 'Propagation of unsteady disturbances in a slowly varying duct with mean swirling flow," Journal of Fluid Mechanics 445, 207-234 (2001).

21 H. Nayfeh, B. S. Shaker, and J. E. Kaiser, "Transmission of sound through nonuniform circular ducts with compressible mean flows," American Institute of Aeronautics and Astronautics Journal 18, 515-525 (1980).

22 K. Uenishi, and M. K. Myers, 'Two-dimensional acoustic field in a nonuniform duct carrying compressible flow," American Institute of Aeronautics and Astronautics Journal 22, 1242-1248 (1984). 
L. Abrahamson, "A finite element algorithm for sound propagation in axisymmetric ducts containing compressible mean flow," AIAA $4^{\text {th }}$ Aeroacoustics Conference, Atlanta (1977).

24 R. J. Astley, and W. Eversman, "A finite element for transmission in non-uniform ducts without flow: comparison with the method of weighted residuals," Journal of Sound and Vibration 57, 367-388 (1978).

25 R. J. Astley, and W. Eversman, "Acoustic transmission in non-uniform ducts with mean flow, part II: the finite element method," Journal of Sound and Vibration 74, 103-121 (1981).

26 R. J. Astley, and W. Eversman, "A finite element formulation of the eigenvalue problem in lined ducts with flow," Journal of Sound and. Vibration $\mathbf{6 5}, 61-74$ (1979).

27 V. Golubev, and H. M. Atassi, "Acoustic-vorticity waves in swirling flows," Journal of Sound and Vibration 209, 203-222 (1998).

L. Greverie, and C. Bailly, 'Construction d'un opérateur de propagation à partir des équations d'Euler linéarisées" ('Formulation of an acoustic wave operator based on linearized Euler equations"), Comptes-rendus de l'Académie des Sciences Série IIb 326, 741-746 (1998).

Longatte, and P. Lafon, 'Computation of acoustic propagation in two-dimensional sheared ducted flow," American Institute of Aeronautics and Astronautics Journal 38, 389-394 (2000).

30 Bailly, and D. Juve, 'Numerical solution of acoustic propagation problems using linearized Euler equations," American Institute of Aeronautics and Astronautics Journal 38, 22-29 (2000). 

Zones de Silence (Propagation of an acoustic wave in the atmosphere and theory of zones of silence) (Gauthier-Villars, Paris, 1931).

32 M. K. Myers, 'On the acoustic boundary condition in the presence of flow," Journal of Sound and Vibration 71, 429-434 (1980).

33 Poiree, 'Les équations de l'acoustique linéaire et non linéaire dans un écoulement de fluide parfait"('Equations of linear and non linear acoustics in a perfect fluid flow'), Acustica 57, 5-25 (1985).

34 O. A. Godin, 'Reciprocity and energy theorems for waves in a compressible inhomogeneous moving fluid," Wave Motion 25, 143-167 (1997).

35 M. Ben Tahar, and E. Goy, 'Resolution of a vibroacoustic problem in the presence of a nonuniform mean flow," Fourth AIAA Joint Aeroacoustics Conference Paper No. 98-2215 (1998).

36 Peyret, and G. Elias, 'Finite-element method to study harmonic aeroacoustics problems," Journal of the Acoustical Society of America 110, 661-668 (2001).

37 S. Bonnet-Ben Dhia, G. Legendre, and E. Luneville, "Analyse mathématique de l'équation de Galbrun en écoulement uniforme" ("Mathematical analysis of Galbrun's equation with uniform flow'), Comptes-rendus de l'Académie des Sciences Série IIb 329, 601-606 (2001).

38 Blokhintzev, 'The propagation of sound in an inhomogeneous and moving medium I," Journal of the Acoustical Society of America 18, 322-328 (1946). 
M. A. Hamdi, and Y. Ousset, "A displacement method for the analysis of vibrations of coupled fluid-structure systems," International Journal for Numerical Methods in Engineering 13, 139-150 (1978).

40 Bermudez, and L. Hervella-Nieto, 'Finite element computation of three-dimensional elastoacoustic vibrations," Journal of Sound and Vibration 219, 279-306 (1999).

41 K. J. Bathe, C. Nitikitpaiboon, and X. Wang, "A mixed displacement-based finite element formulation for acoustic fluid-structure interaction," Computer and Structures 56, 225-237 (1995).

42 X. Wang, and K. J. Bathe, 'Displacement/Pressure based mixed finite element formulations for acoustic fluid-structure interaction problems," International Journal for Numerical Methods in Engineering 40, 2001-2017 (1997).

43 K. J. Bathe, Finite Element Procedures (Prentice Hall, Englewood Cliffs, 1996).

44 J.-M. Jin, The Finite Element Method in Electromagnetics (John Wiley \& Sons Inc., New York, 1993).

45 F. Brezzi, and M. Fortin, Mixed and Hybrid Finite Element Methods (SpringerVerlag, New York, 1991).

46 D. Chapelle, and K. J. Bathe, "The inf-sup test," Computer and Structures 47, 537545 (1993). 


\section{FIGURE CAPTIONS:}

FIG.1: Typical geometry of a duct carrying flow $\left(S_{1}\right.$ denotes the wall boundaries and $S_{2}$ the inlet and outlet boundaries).

FIG.2: Examples of 2-D elements satisfying the inf-sup condition. (a) Element "4-3c" (used in this paper), which may be referred to as the " $P_{l}{ }^{+}-P_{l}$ " or "MINI" element in the literature. (b) Example of higher order element, with 9 nodes for the displacement and 4 nodes for the pressure (element ' $9-4 c$ ').

FIG.3: Pressure modulus in $\mathrm{Pa}$ of the $(0,1)$ mode at $f=250 \mathrm{~Hz}$ (no flow and rigid walls).

(a) semi-analytical solution, (b) displacement-based FEM solution, (c) mixed FEM solution, (d) FEM mesh.

FIG.4: Pressure modulus in $\mathrm{Pa}$ of the $(8,0)$ mode at $f=500 \mathrm{~Hz}$ (rigid walls). (a) PridmoreBrown and (b) mixed FEM solutions with $M=0.0$. (c) Pridmore-Brown and (d) mixed FEM solutions with $M=-0.4$. (e)-(f) intensity vector (computed from the FEM model) for $M=0.0$ and $M=-0.4$ respectively.

FIG.5: Pressure phase in radians of the $(8,0)$ mode at $f=500 \mathrm{~Hz}$ with $M=-0.4$ (rigid walls). (a) Pridmore-Brown and (b) mixed FEM solutions. 
FIG.6: Pressure modulus in Pa of the $(10,1)$ mode at $f=1000 \mathrm{~Hz}$ and $M=-0.5$ with lined walls $(Z=2040-2040 i)$. (a) Pridmore-Brown and (b) mixed FEM solutions for a uniform flow. (c) Pridmore-Brown and (d) mixed FEM solutions with a boundary layer thickness of $10 \%$.

FIG.7: Axial velocity profiles for the shear and uniform mean flows. Both flows have the same mass flow rates (the mean Mach number is 0.5 ).

FIG.8: Rienstra's duct geometry with a straight outer wall. (a) FEM mesh, (b) radial and (c) axial potential mean flow velocities (in Mach number) computed from a FEM model of Laplace's equation.

FIG.9: Pressure modulus in Pa of the $(1,1)$ mode (rigid walls). (a)-(b)-(c) multiple-scales semi-analytical solutions at $f=300,360$, and $420 \mathrm{~Hz}$ respectively, (d)-(e)-(f) mixed FEM solutions at $f=300,360$, and $420 \mathrm{~Hz}$.

FIG.10: Pressure modulus in $\mathrm{Pa}$ of the $(7,0)$ mode at $500 \mathrm{~Hz}$ with lined walls ( $Z=4080+4080 i)$ for the exact Rienstra's duct geometry. (a)-(b) comparison between multiple-scales and mixed FEM solutions for a downstream propagation. (c)-(d) comparison for an upstream propagation. 
F. Treyssède, JASA

FIG.1

Fabien Treyssède

JASA

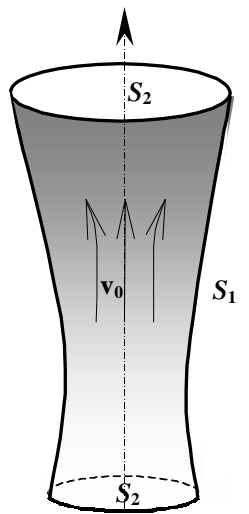


F. Treyssède, JASA

FIG.2

Fabien Treyssède

JASA

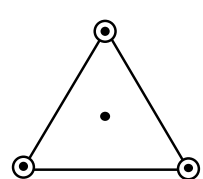

(a)



(b)

- Displacement degrees of freedom

Pressure degrees of freedom 
FIG.3

Fabien Treyssède

JASA

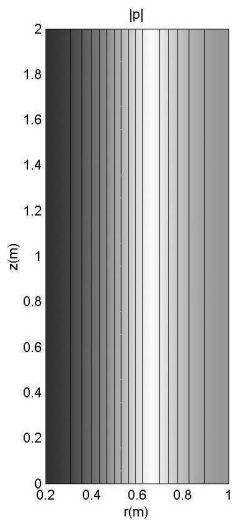

(a)

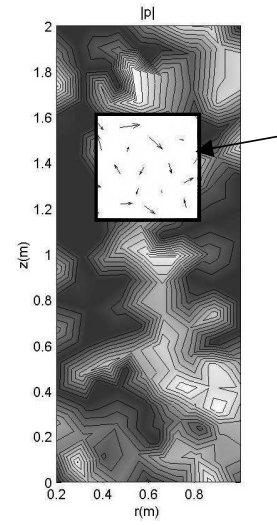

(b)



(c)

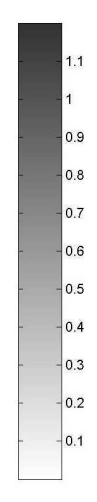

\{

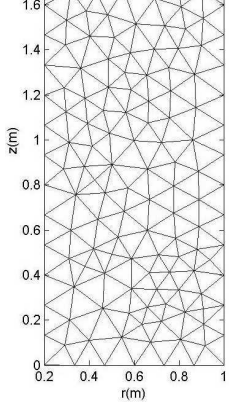

(d) 
F. Treyssède, JASA

\section{FIG.4}

Fabien Treyssède

JASA

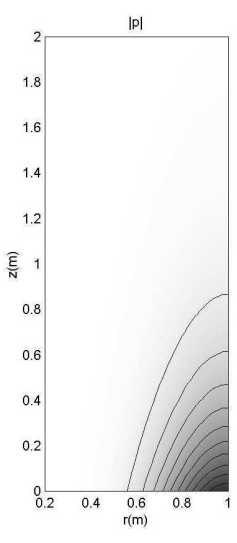

(a)

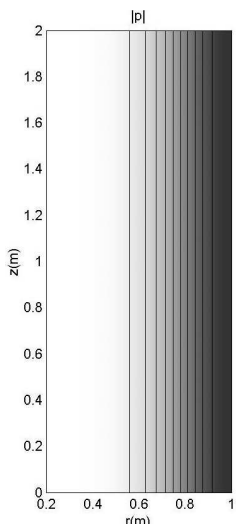

(c)

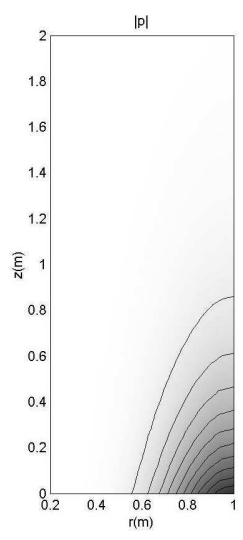

(b)

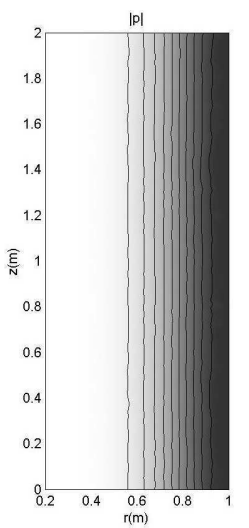

(d)
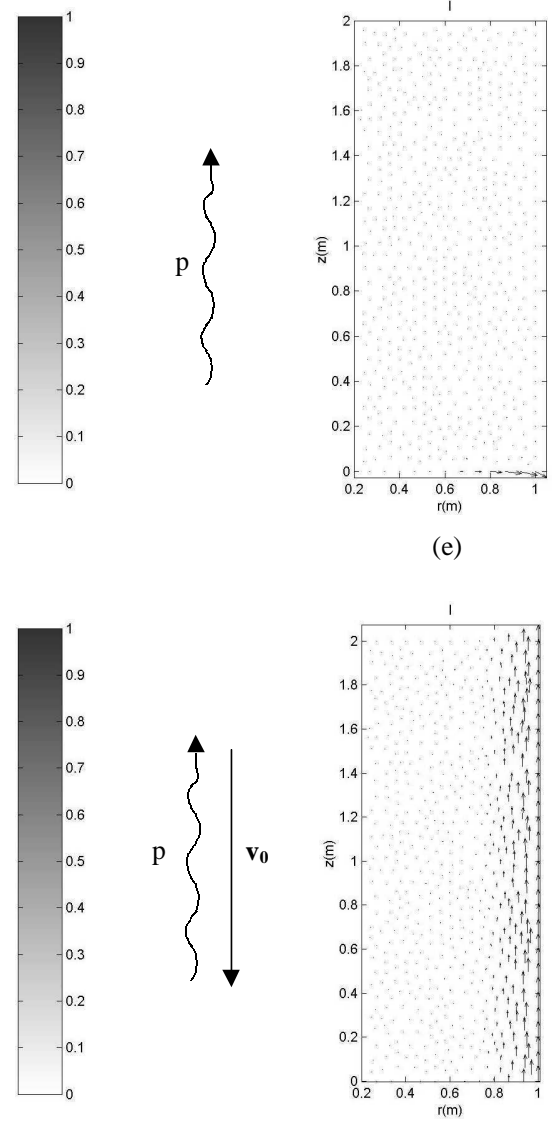

(f) 


\section{FIG.5}

Fabien Treyssède

JASA

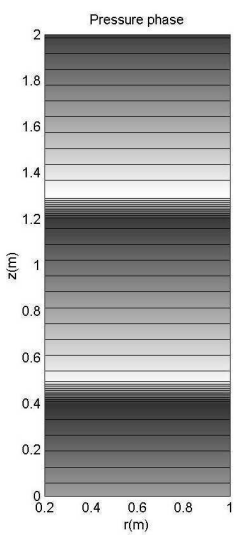

(a)

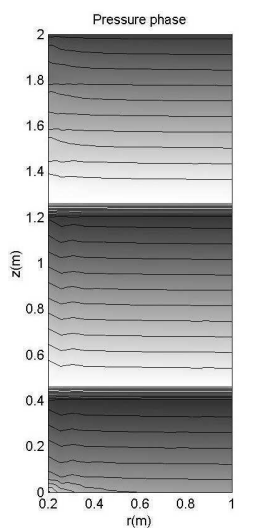

(b)
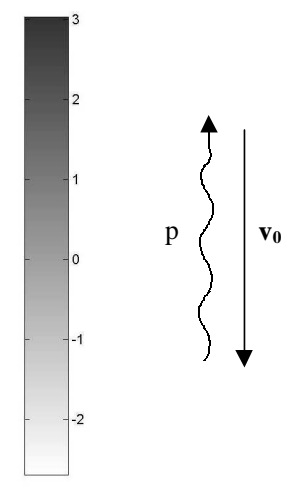
FIG.6

Fabien Treyssède

JASA

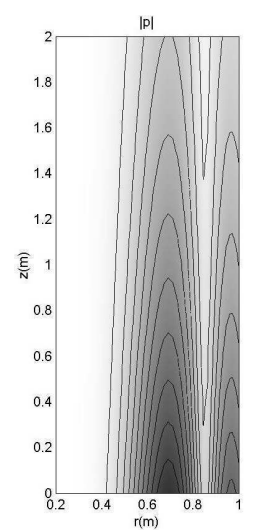

(a)

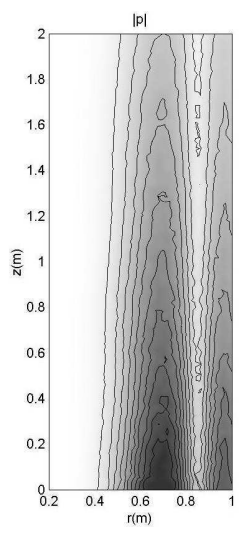

(b)

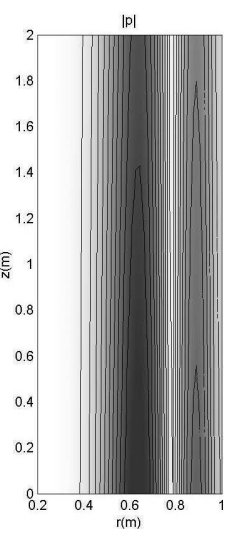

(c)

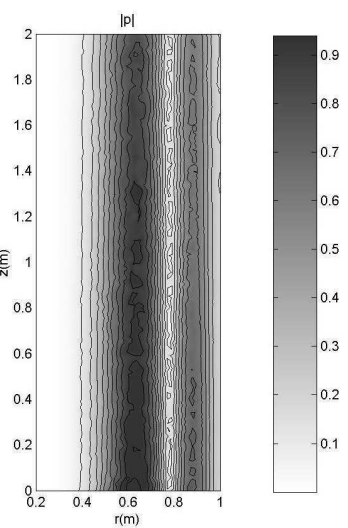

(d)




F. Treyssède, JASA

FIG.7

Fabien Treyssède

JASA

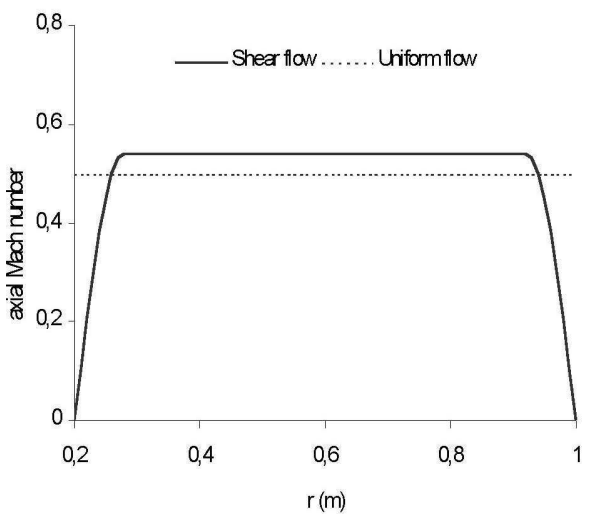


FIG.8

Fabien Treyssède

JASA

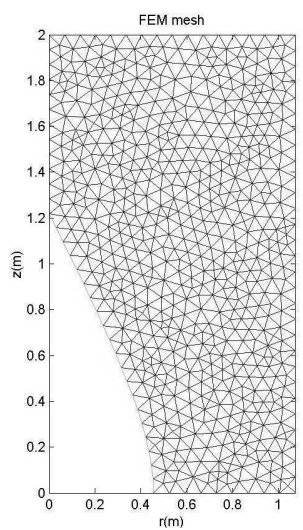

(a)

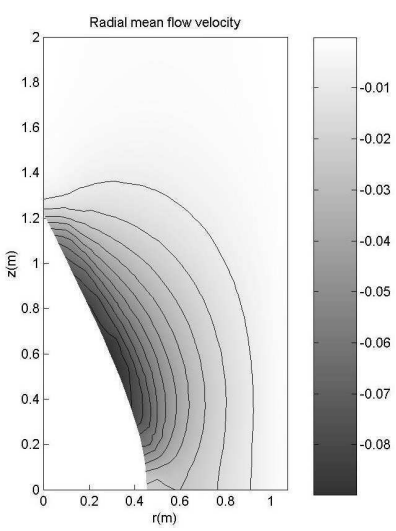

(b)

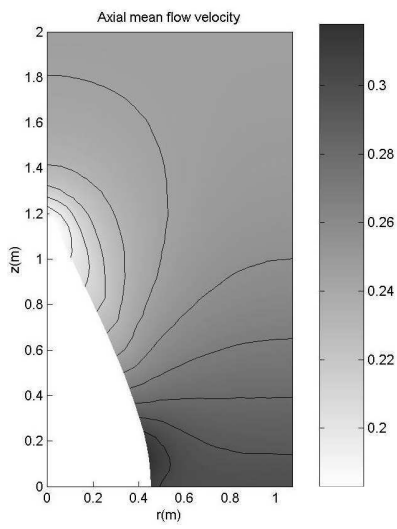

(c) 


\section{FIG.9}

Fabien Treyssède

JASA

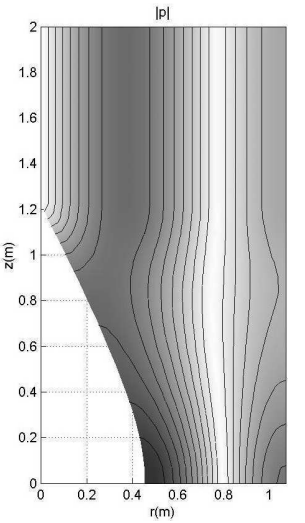

(a)

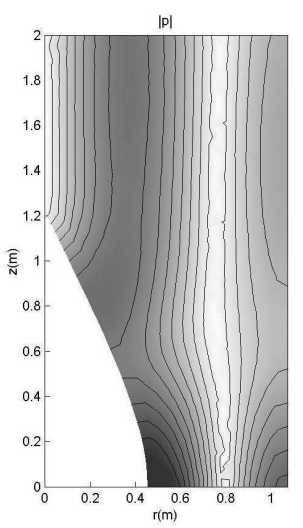

(d)

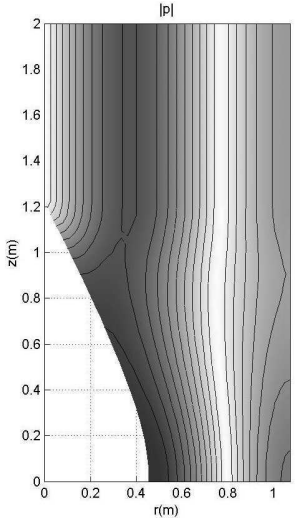

(b)

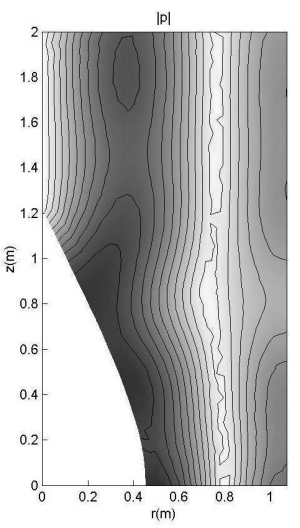

(e)

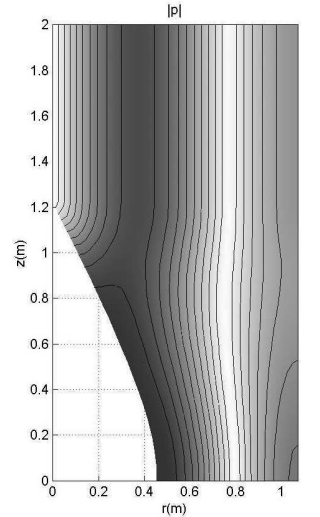

(c)



(f)
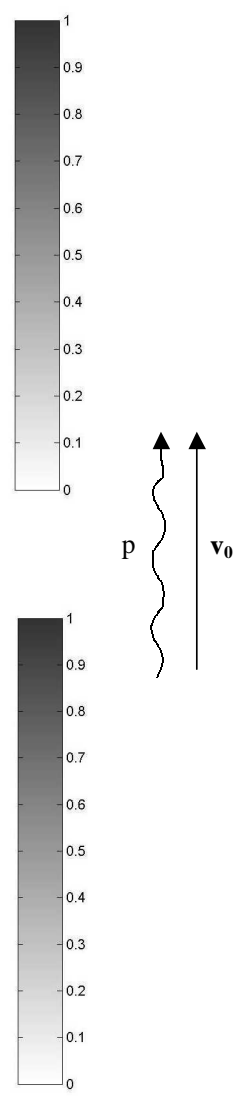
FIG.10

Fabien Treyssède

JASA

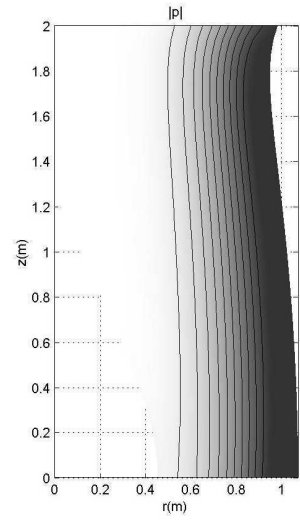

(a)

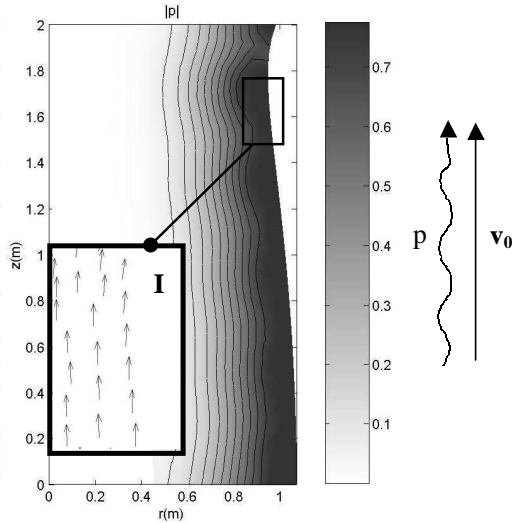

(b)

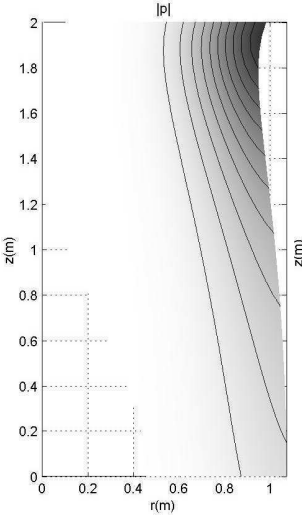

(c)

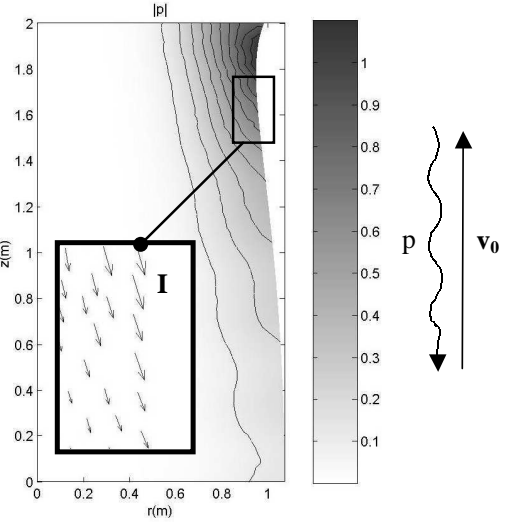

(d) 